\title{
Influência da doença de Parkinson em capacidade física, função pulmonar e índice de massa magra corporal
}

\author{
Influence of the Parkinson's disease on physical \\ capacity, lung function and lean body mass index
}

\section{Lélia Arantes Bonjorni ${ }^{[a]}$, Mauricio Jamami ${ }^{[b]}$, Valéria Amorim Pires Di Lorenzo ${ }^{[b]}$, Bruna Varanda Pessoa ${ }^{[\mathrm{c}]}$}

[a] Fisioterapeuta, aluna do curso de Especialização em Fisioterapia Geriátrica da Universidade Federal de São Carlos (UFSCar), São Carlos, SP - Brasil, e-mail: leliabonjorni@gmail.com

[b] Professores doutores adjuntos do curso de Graduação em Fisioterapia e do Programa de Pós-Graduação em Fisioterapia, Laboratório de Espirometria e Fisioterapia Respiratória, Departamento de Fisioterapia - Universidade Federal de São Carlos (UFSCar), São Carlos, SP - Brasil, e-mails: jamami@ufscar.br; vallorenzo@ufscar.br

[c] Doutoranda do Programa de Pós-Graduação em Fisioterapia da Universidade Federal de São Carlos (UFSCar), São Carlos, SP - Brasil, e-mail: brunavpessoa@gmail.com

\section{Resumo}

Introdução: Pacientes com Doença de Parkinson (DP) apresentam obstrução grave das vias aéreas, fadiga e incoordenação dos músculos respiratórios, redução de massa muscular e fraqueza e fadiga musculares, levando a intolerância ao exercício e piora da qualidade de vida. Objetivos: Avaliar e comparar o índice de massa magra corporal (IMMC), variáveis espirométricas, força muscular respiratória e capacidade física, em pacientes com DP e idosos saudáveis. Além disso, verificar quais dessas variáveis influenciam a capacidade física de pacientes com DP. Materiais e métodos: Foram avaliados 25 indivíduos, de ambos os sexos, sendo dez pacientes com DP (Grupo DP [GDP]: 72,7 \pm 10,0 anos) e 15 idosos saudáveis (Grupo saudável [GS]: 64,8 \pm 6,7 anos), pela espirometria, pressões inspiratória (PImáx) e expiratória máximas (PEmáx), composição corporal e do teste de caminhada de seis minutos (TC6). Resultados: O GDP apresentou valores significativamente menores de relação volume expiratório forçado no primeiro segundo/capacidade vital forçada, ventilação voluntária máxima (VVM), massa muscular, PImáx, PEmáx, e distância percorrida no TC6 comparado ao GS. No GDP, observaram-se correlações positivas moderadas significativas da PEmáx e VVM com a distância percorrida no TC6 ( $r=0,84 ; r=0,67$, respectivamente), entre o IMMC com VVM ( $r=0,69)$. Conclusão: O GDP apresentaram 
redução da massa muscular, da endurance e forças musculares respiratórias, e da capacidade física, se comparados aos idosos saudáveis. Além disso, a PEmáx e a VVM influenciam a capacidade física medida pelo TC6, assim como a massa muscular influencia a endurance da musculatura respiratória nos pacientes com DP. Assim, devemos tornar o TC6 um método padrão de avaliação na prática clínica.

Palavras-chave: Doença de Parkinson. Idoso. Esforço físico.

\section{Abstract}

Introduction: Patients with Parkinson's disease (PD) have severe airway obstruction, fatigue and respiratory muscle incoordination, reduced muscle mass and muscle weakness and fatigue, leading to exercise intolerance and reduced quality of life. Objectives: To assess and compare the lean body mass index (LBMI), spirometric variables, respiratory muscle strength and physical capacity of DP patients and healthy elderly. Also, verify which of these variables influence exercise capacity in patients with PD. Materials and methods: Twenty-five individuals of both sexes were evaluated: ten DP patients (PD group [PDG]: $72.7 \pm 10.0$ years old) and 15 healthy elderly (Healthy group [HG]: $64.8 \pm 6.7$ years old), through spirometry, respiratory muscle strength, body composition and 6MWT. Results: The PDG presented significantly lower values, forced expiratory volume in one second/forced vital capacity, maximum voluntary ventilation (MVV), muscle mass, maximal inspiratory (MIP) and expiratory pressures (MEP) and the covered distance in the 6MWT compared to HG. In PDG, moderately positive correlations significant $(p<0.001)$ were observed between MEP and MVV with covered distance in the 6MWT $(r=0.84 ; r=0.67$, respectively), and between the LBMI with MVV ( $r=0.69)$. Conclusion: The patients of PDG had reduced muscle mass, respiratory muscle strength and endurance, and of physical capacity compared to healthy elderly. Also, expiratory muscle strength and respiratory muscles endurance influence physical capacity (measured by 6MWT), as well as muscle mass influence the respiratory muscle endurance in patients with PD. This way, we have found the importance of the 6MWT to become a standard method of assessment in clinical practice in patients with DP.

Keywords: Parkinson's disease. Elderly. Physical exertion.

\section{Introdução}

A Doença de Parkinson (DP) tem característica crônica e progressiva e acomete, em geral, a população idosa na faixa etária entre 50 a 70 anos, atingindo um pico entre os 60 anos e aumentando tanto a prevalência quanto a incidência de DP. Aproximadamente 1 a $2 \%$ da população brasileira acima de 65 anos apresenta a DP $(1,2)$.

Com o envelhecimento fisiológico, ocorre uma diminuição gradativa de massa corporal, função pulmonar, força e endurance muscular respiratória e periférica, e da capacidade física, levando aos efeitos debilitantes (3). Quando associados à DP, observa-se uma crescente perda da funcionalidade, afetando ainda mais o estado nutricional, piorando a qualidade de vida e a independência funcional do indivíduo (4).

A função respiratória é afetada diretamente pela doença, e em estágios avançados da DP, constata-se obstrução grave das vias aéreas superiores, sendo esta a maior anormalidade pulmonar. Além disso, diminuição da complacência da caixa torácica, diminuição do volume de ar e das taxas de fluxo de ar inspirado, fadiga e incoordenação dos músculos diafragmáticos e acessórios, o que funciona como um grande indicador de doença neuromuscular (4- 7).

Esses pacientes apresentam redução significativa das fibras musculares do tipo IIA, levando à atrofia por desuso ou descondicionamento físico (8), predispondo à fraqueza e fadiga musculares. Ainda, a redução de massa muscular leva a incoordenação dos movimentos, quedas frequentes, intolerância ao exercício e piora progressiva do condicionamento físico, limitando as atividades de vida diária (7-12).

0 teste de caminhada de seis minutos (TC6) é considerado uma das formas alternativas de avaliação da capacidade física, por ser submáximo $(13,14)$, ser opção de fácil realização, baixa complexidade, além de não requerer equipamentos de alto custo na prática clínica $(14,15)$.

Assim, faz-se necessária uma avaliação minuciosa, visto que esses pacientes apresentam intolerância ao 
esforço físico, levando ao comprometimento na execução de simples atividades diárias, como a caminhada, além de ser importante para estabelecer um programa de tratamento fisioterapêutico adequado às limitações e à gravidade da doença. Isso porque são escassos os estudos analisando as variáveis que influenciam a capacidade física de pacientes com DP, avaliada pelo TC6.

Sendo assim, os objetivos do presente estudo foram avaliar e comparar índice de massa magra corporal (IMMC), variáveis espirométricas, força muscular respiratória e capacidade física entre pacientes com DP e idosos saudáveis. Além disso, verificar quais dessas variáveis influenciam a capacidade física de pacientes com DP.

\section{Materiais e métodos}

\section{Casuística}

Este estudo foi realizado no Laboratório de Espirometria e Fisioterapia Respiratória e no Laboratório de Neurologia da Universidade Federal de São Carlos (UFSCar). Foram avaliados 25 indivíduos, na faixa etária de 55 a 82 anos, de ambos os sexos: dez pacientes portadores de DP encaminhados ao setor de fisioterapia e cadastrados na Unidade Saúde Escola (USE) da UFSCar, compondo o grupo com DP (GDP); e 15 indivíduos idosos saudáveis recrutados na comunidade de São Carlos (SP) e região, compondo o grupo saudável (GS).

\section{Critérios de inclusão}

Foram adotados como critérios de inclusão para o GDP: pacientes na faixa etária entre 55 e 82 anos; que estivessem realizando fisioterapia convencional; não apresentassem complicações ortopédicas impossibilitando a realização do protocolo; não apresentassem alterações cognitivas graves; apresentassem grau de acometimento da DP entre um e quatro na escala de Hoehn e Yahr versão modificada (16), e não sofressem com doenças respiratórias prévias. Para o GS, os critérios de inclusão foram: indivíduos não fumantes que apresentassem funções espirométricas dentro dos padrões de normalidade; não apresentassem doença neurológica e/ou respiratória; estivessem na faixa etária de 55 e 82 anos; não tivessem participado de protocolo de treinamento físico nos últimos três meses anteriores ao início do estudo; fossem classificados como sedentários ou insuficientemente ativos de acordo com o Questionário Internacional de Atividade Física - versão curta (IPAQ) (17).

Todos assinaram o termo de consentimento livre e esclarecido mediante orientações sobre o protocolo proposto, e o estudo foi aprovado pelo Comitê de Ética da UFSCar, Parecer n. 313/2011.

\section{Procedimento experimental}

Os indivíduos do GDP e GS foram submetidos à anamnese, avaliação física geral, além de espirometria, avaliação da força muscular respiratória, da composição corporal e da capacidade física pelo teste de caminhada de seis minutos (TC6) - realizados nessa ordem.

\section{Espirometria}

Foi realizada por meio de um espirômetro portátil (Micro Quark PC - Based Espirometer ${ }^{\circledR}$ ), de acordo com os procedimentos técnicos, critérios de aceitabilidade e reprodutibilidade, segundo as normas da American Thoracic Society/European Respiratory Society (ATS/ERS) (18).

\section{Avaliação da composição corporal}

Foi realizada por meio de uma balança de impedância bioelétrica de tecnologia bipolar (Tanita ${ }^{\circledR}$, modelo BC-553, Illinois, USA), devendo os pacientes estar de jejum por, pelo menos, quatro horas para padronização de ingestão líquida, e vestindo roupas leves. As medidas foram obtidas com os pacientes na posição ortostática, com seus calcanhares devidamente alinhados aos eletrodos da balança, obtendo-se as medidas de massa corporal total e massa magra muscular (19). Posteriormente, foi calculado o índice de massa magra corporal (IMMC; índice de depleção nutricional): massa muscular $(\mathrm{kg}) /$ altura $^{2}(\mathrm{~m})(20)$, considerando depleção nutricional, homens com IMMC $<16,0 \mathrm{Kg} / \mathrm{m}^{2}$ e mulheres com IMMC $<15,0 \mathrm{Kg} / \mathrm{m}^{2}(20)$.

Avaliação da força da musculatura respiratória

A força muscular respiratória foi obtida pela mensuração das pressões inspiratórias e expiratórias máximas (PImáx e PEmáx) com o sujeito em 
posição ortostática, utilizando um manovacuômetro analógico (Ger-Ar Famabras ${ }^{\circledR}$ ) com limite operacional de $-300 \mathrm{a}+300 \mathrm{cmH}_{2} \mathrm{O}$; durante as manobras, foi usado um clipe nasal a fim de evitar o escape de ar pelo nariz (21). Em cada manobra, o indivíduo realizou um esforço respiratório máximo mantido por pelo menos um segundo, com incentivo verbal por parte do avaliador. As manobras de PImáx e PEmáx foram realizadas no mínimo três e no máximo seis vezes, para eliminar o efeito da aprendizagem e até obter valores coerentes entre si, isto é, com diferença menor que $10 \%$, sendo considerado para a análise estatística o maior valor obtido. Cada indivíduo foi avaliado pelo mesmo examinador em todas as etapas.

Teste de caminhada de seis minutos

0 TC6 foi realizado em um corredor plano e coberto, com 30 metros de comprimento e 1,5 metros de largura, com demarcações a cada dois metros em uma área externa, e sem acompanhamento, seguindo princípios da ATS (13). Os indivíduos foram orientados a caminhar o mais rápido possível durante seis minutos e incentivados com frases preestabelecidas, em intervalos padronizados de um minuto, sendo livre a cadência dos testes. 0 desempenho físico no TC6 foi determinado pela distância total percorrida em metros, e posteriormente calculada a distância prevista por Iwama et al. (22).

Foram registradas no repouso (basal), no segundo, quarto e sexto minutos e no primeiro, terceiro e sexto minutos da recuperação do TC6, a saturação periférica de oxigênio $\left(\mathrm{SpO}_{2}\right)$ por meio de um oxímetro de pulso portátil (Nonin ${ }^{\circledR}$, modelo 2500, Minneapolis, Mn, USA), a frequência cardíaca por meio de um frequencímetro de pulso (Polar Vantage $\mathrm{NVTM}^{\circledR}$, modelo 1901001, Kempele, Oulu, Finlândia), e os sintomas de dispneia e fadiga de membros inferiores pela escala de Borg modificada CR10 (23). As medidas da pressão arterial, obtidas com o indivíduo na posição sentada e com um esfigmomanômetro de coluna de mercúrio (Oxigel ${ }^{\circledR}$, São Paulo, SP, Brasil), foram verificadas no repouso, imediatamente após os TC6 e no primeiro, terceiro e sexto minutos da recuperação.

\section{Análise estatística}

Os resultados desta investigação foram analisados pelo pacote estatístico Statistical Package for
Social Sciences para Windows, versão 18.0 (SPSS Inc, Chicago, IL). A distribuição dos dados foi verificada pelo teste de normalidade de Shapiro-Wilk. Os dados foram expressos em médias \pm desvios padrão. Para a análise intergrupos, utilizou-se o teste $t$ não pareado para todas as variáveis analisadas, e o coeficiente de correlação de Pearson.

O cálculo do poder amostral foi realizado pelo programa GraphPad StatMate 2.0 (San Diego, CA, USA) para Windows, baseado em um estudo piloto no desvio padrão e na diferença das médias das variáveis analisadas, correspondendo a um poder acima de $80 \%$. 0 nível de significância adotado foi de 5\%.

\section{Resultados}

Cinco pacientes do GDP apresentavam obstrução pulmonar leve e cinco apresentavam obstrução pulmonar moderada, segundo a classificação da gravidade proposta pelo GOLD (24). Verificou-se, também, que em ambos os grupos (GDP e GS), dois indivíduos do sexo masculino apresentaram depleção nutricional (IMMC $\left.<16,0 \mathrm{Kg} / \mathrm{m}^{2}\right)$ (20).

Quanto às variáveis antropométricas, na análise intergrupos, não foram observadas diferenças estatisticamente significativas, atestando, assim, a homogeneidade da amostra, exceto para a variável massa muscular ( $p<0,001)$, sendo esta significativamente menor para o GDP.

$\mathrm{Na}$ análise intergrupos, observou-se diferença estatisticamente significativa em relação aos valores espirométricos: relação $\mathrm{VEF}_{1} / \mathrm{CVF}(\mathrm{p}<0,0001)$ e ventilação voluntária máxima (VVM) $(p<0,004)$, a PImáx $(\mathrm{p}<0,04)$ e PEmáx $(\mathrm{p}<0,03)$, e quanto à capacidade física medida pela distância percorrida no TC6 ( $p<0,0001)$, valores significativamente menores para o GDP se comparado ao GC.

Não foram constatadas diferenças significativas quanto ao IMMC, e variáveis espirométricas: $\mathrm{VEF}_{1} \mathrm{e}$ CVF, entre os grupos.

Observamos correlações positivas moderadas estatisticamente significativas no GDP entre a PEmáx e a VVM com distância percorrida no TC6 $(\mathrm{r}=0,84 ; \mathrm{p}<0,008 ;$ e $\mathrm{r}=0,67 ; \mathrm{p}<0,04$, respectivamente), e entre o IMMC com a VVM ( $r=0,69$; $\mathrm{p}<0,002$ ). As Figuras $1 \mathrm{~A}, 1 \mathrm{~B}$ e $1 \mathrm{C}$ mostram as correlações entre a distância percorrida no TC6 com a PEmáx e a VVM, e a correlação entre o IMMC e a VVM nos pacientes com DP. 
Quadro 1 - Características demográficas, antropométricas, espirométricas, força muscular respiratória e capacidade física no TC6 do GDP e GS

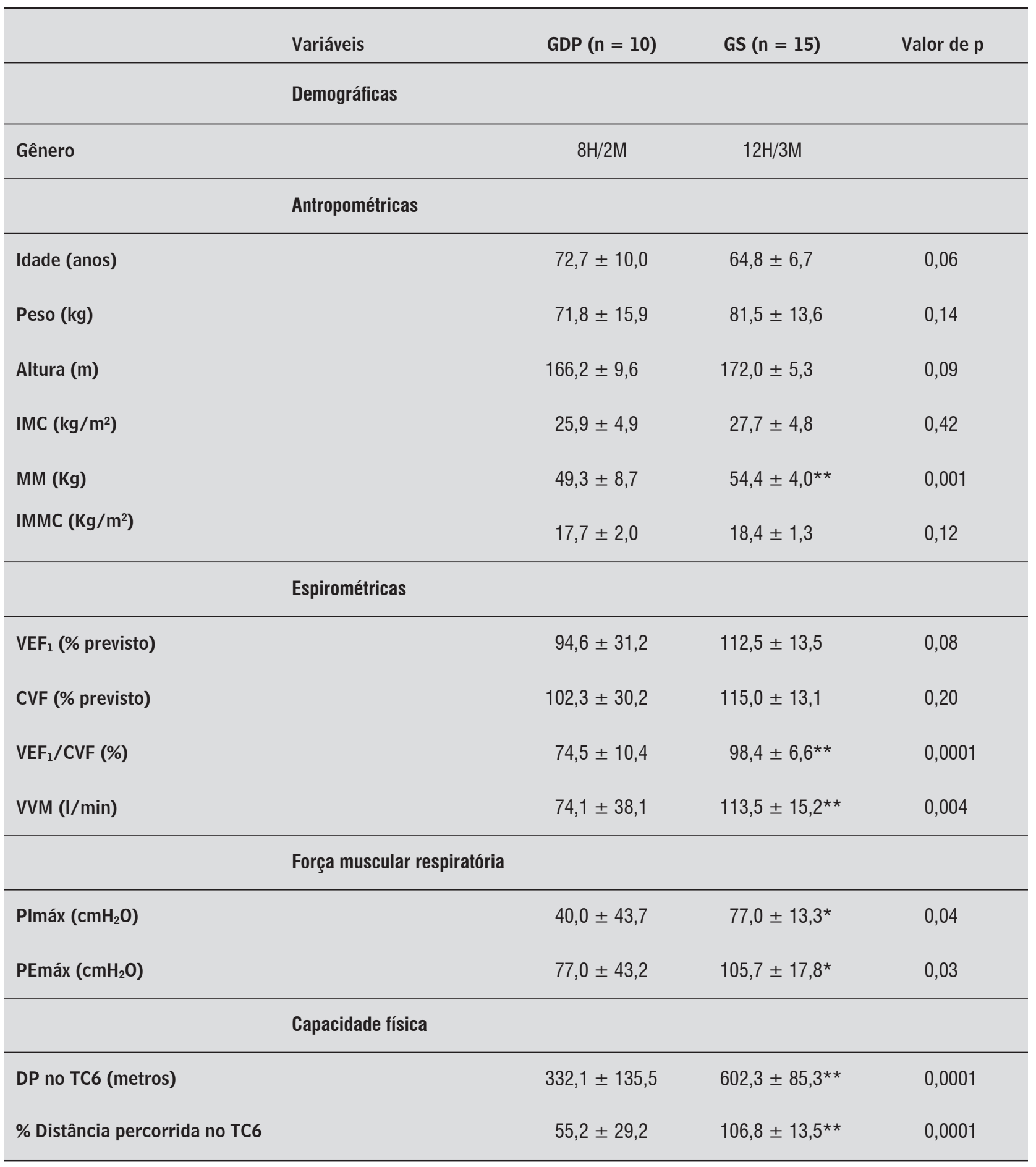

Legenda: GDP = grupo doença de Parkinson; GS = grupo saudável; IMC = índice de massa corporal; $M M M=$ massa muscular magra; IMMC = índice de massa magra corporal; $\mathrm{VEF}_{1}=$ volume expiratório forçado no primeiro segundo; $\mathrm{CVF}=$ capacidade vital forçada; $\mathrm{VEF}_{1} /$ CVF = relação VEF $/$ /CVF; VVM = ventilação voluntária máxima; PImáx = pressão inspiratória máxima; PEmáx = pressão inspiratória máxima; DP = distância percorrida; TC6 = teste de caminhada de seis minutos em corredor.

Fonte: Dados da pesquisa.

Nota: valores expressos em médias e desvios padrão; ${ }^{*}=$ Diferença significativa entre os grupos GDP versus CG; ${ }^{*}=p<0,05 ;{ }^{* *}=$ $p<0,001$. 


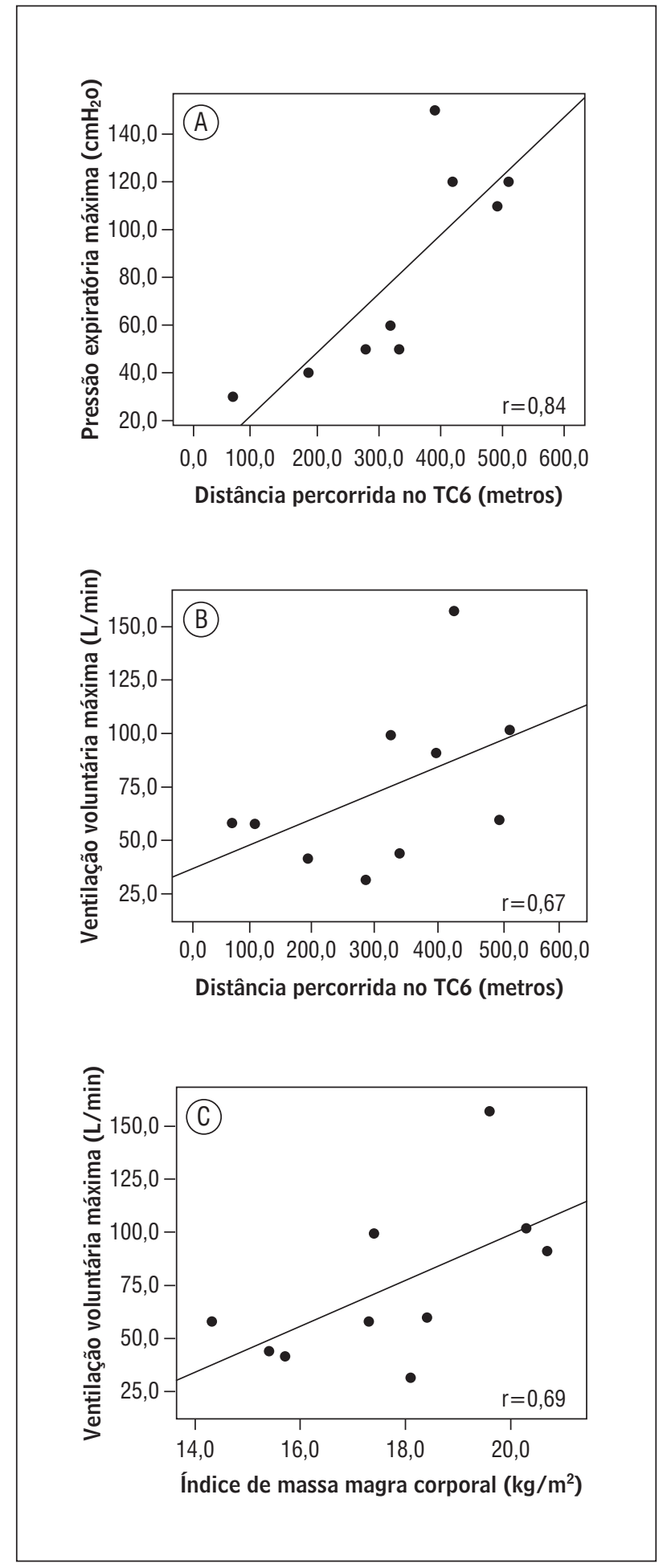

Figuras 1A, 1B e 1C - Correlação entre a distância percorrida no teste de caminhada de seis minutos (TC6) e a pressão expiratória máxima e a ventilação voluntária máxima, e da ventilação voluntária máxima e 0 índice de massa magra corporal nos pacientes com DP, respectivamente

Fonte: Dados da pesquisa.

\section{Discussão}

Os principais achados do presente estudo podem ser assim sumarizados: os indivíduos do GDP apresentaram diminuição da massa muscular magra, da relação $\mathrm{VEF}_{1} / \mathrm{CVF}$, das forças musculares respiratórias, da endurance da musculatura respiratória e da capacidade física medida pela distância percorrida no TC6, se comparados aos do GS. Além disso, notamos correlação entre a PEmáx e a VVM com a capacidade física (TC6), e entre o IMMC e a VVM no GDP.

Sabe-se que a função pulmonar deteriora-se gradualmente ao longo da vida adulta, com declínio progressivo do $\mathrm{VEF}_{1}$ e da CVF, independentemente do tabagismo e da exposição ambiental, estando associada ao aumento da morbidade e da mortalidade (25).

No GDP, observou-se diminuição da relação $\mathrm{VEF}_{1}$ / CVF caracterizando a obstrução das vias aéreas e diminuição do $\mathrm{VEF}_{1}$ quando comparado ao GS, porém a diminuição do $\mathrm{VEF}_{1}$ não foi considerada significativa $(p=0,08)$. Acredita-se que isso se deve ao número reduzido de pacientes no GDP, e também por cinco pacientes apresentarem obstrução leve das vias aéreas e cinco obstruções moderadas, e por não apresentar nenhum paciente com obstrução grave. Esses resultados são contrários aos de Alves, Coelho e Brunetto (5), que relatam que, após cinco meses (40 sessões) de intervenção fisioterapêutica, observaram quadro restritivo e aumento do $\mathrm{VEF}_{1}, \mathrm{CVF}$, pico de fluxo expiratório e da VVM, traduzindo em melhora (média de 19\%) da expansão torácica, com consequente aumento dos volumes e fluxos pulmonares; Sathyaprabha et al. (7) concluíram que a disfunção pulmonar restritiva está presente nos pacientes com DP, a qual melhora significativamente com administração da levodopa. Os resultados deste estudo corroboram com três estudos registrados durante o ano de 1960, os quais sugeriram que os pacientes com DP apresentam disfunção pulmonar obstrutiva (25-29).

Sabe-se que a VVM é uma importante ferramenta de avaliação e evidencia o grau de limitação ventilatória nos pacientes com doença pulmonar obstrutiva crônica (DPOC) (30). Na DP é considerada importante, pois demostra a dificuldade de coordenação muscular para a realização de movimento rápido e repetitivo (5), além de ser um indicador de doença neuromuscular, e sua diminuição está associada à bradicinesia e rigidez da caixa torácica, sintomas da DP (7).

Além disso, o GDP apresentou diminuição das forças musculares respiratórias inspiratórias e expira- 
tórias, e da endurance da musculatura respiratória, se comparado ao GS - explicada pela característica fisiológica do envelhecimento associada a característica patofisiológica da DP $(6,26)$-, corroborando com os achados de Alves, Coelho e Brunetto (5) e Sabaté et al. (26). Sugere-se que isso se deve ao aumento da rigidez da caixa torácica, redução da pressão de recolhimento elástico, diminuição do volume de ar e das taxas de fluxo de ar inspirado, além da fadiga e incoordenação dos músculos diafragmáticos e acessórios, sendo isso um grande indicador de doença neuromuscular $(4,5,6,7)$.

No que se refere à composição corporal, a variável massa magra apresentou valores menores nos pacientes com DP em relação aos indivíduos do GS, fato atribuído à atrofia por desuso ou descondicionamento físico (31), causada pelas manifestações da DP. Nos idosos, com o envelhecimento ocorre uma redução das fibras musculares do tipo IIA, levando à atrofia, predispondo à fraqueza e fadiga musculares e à redução de massa muscular, diminuindo o potencial muscular e a endurance muscular periférica, provocando incoordenação dos movimentos, quedas frequentes e intolerância ao exercício, aumentando o declínio funcional, bem como a morbidade e a mortalidade $(7,9,10)$.

A capacidade física, a distância percorrida no TC6 e a porcentagem da distância percorrida apresentaram-se menores para o GDP se comparadas às do GS, verificando uma redução de aproximadamente $270 \mathrm{~m}$, sendo considerado um fator clinicamente importante e significativo (32). Também observamos que os indivíduos do GDP e GS atingiram 55,2\% e 106,8\% da distância prevista para o TC6, respectivamente, segundo Iwana et al. (22). Atribui-se essa diminuição à fraqueza muscular periférica nos pacientes do GDP, pois ela foi descrita previamente como um importante fator na determinação da capacidade física no TC6 em pacientes com DPOC (33). Sabe-se que a fraqueza muscular é proporcional à perda de massa muscular (34), havendo evidências de que pacientes com DPOC apresentam redução significativa da força de membros inferiores quando comparados a congêneres controles $(35,36)$. No entanto, a redução da força muscular é predominante nos membros inferiores, e tal fato faz com que os pacientes com DPOC evitem as atividades que envolvem a marcha, em virtude da dispneia $(34,35)$.

Os pacientes do GDP caminharam aproximadamente $332,1 \pm 135,5 \mathrm{~m}$, e os pacientes com DP do estudo de Falvo e Earhart (37) caminharam 391,6 \pm 99,9 m. Assim, esses autores concluíram que os indivíduos com DP de estágio leve a moderado apresentam melhor prognóstico para a capacidade de exercício normal com exercício aeróbio regular. O GDP deste trabalho não realizou exercício físico aeróbio regular.

Observaram-se correlações positivas moderadas no GDP entre a PEmáx e a VVM com a distância percorrida no TC6, e do IMMC com a VVM, sugerindo uma relação significativa que quanto maior a força muscular expiratória e a endurance muscular respiratória, maior a capacidade física; e quanto maior a quantidade de massa muscular, maior a endurance muscular respiratória nesses pacientes, resultados não encontrados na literatura científica para a população estudada. Falvo e Earhart (37) verificaram correlação moderada negativa entre distância percorrida no TC6 e o Timed Up \& Go (TUG), mostrando que aproximadamente 55\% da variação da distância percorrida no TC6 nos indivíduos com DP pode ser um fator específico para a característica da DP e prejuízo em equilíbrio e mobilidade, identificando maior influência na capacidade física.

Não encontramos estudos na literatura que demonstrem o que encontramos quanto à correlação entre a distância percorrida no TC6 e a VVM nos pacientes com DP. Entretanto, Pitta et al. (27), Brunetto et al. (30) e Cestaro et al. (34) mostraram que há relação de uma atividade que simula uma atividade física de vida diária (caminhada) com a resistência da musculatura respiratória nos indivíduos com DPOC, verificando que os pacientes com DPOC que apresentaram maior VVM ao repouso percorreram maior distância no TC6, porém esses achados não foram encontrados para a população de pacientes com DP.

Assim, concluímos que a redução significativa das fibras musculares do tipo IIA presente nos pacientes com DP (8), que predispõe à fraqueza muscular periférica e respiratória, e a diminuição da VVM, associada à bradicinesia e rigidez da caixa torácica, bem como a redução de massa muscular, que gera incoordenação dos movimentos e quedas frequentes, resultam na intolerância ao exercício nos pacientes com DP (7-12).

Optou-se por utilizar o TC6 para avaliar a capacidade física de pacientes com DP, por ser uma das formas alternativas de avaliação aos testes máximos, por ser submáximo (14), e por mostrar ser uma opção de fácil realização, baixa complexidade, além de apresentar boa reprodutibilidade e confiabilidade, 
e por não requerer equipamentos de alto custo na prática clínica $(14,15)$. Além disso, trata-se de um preditor de morbidade e mortalidade (38-40), e reflete a capacidade funcional de pacientes com DPOC ao realizar as atividades físicas da vida diária (31).

\section{Considerações finais}

Os indivíduos com doença de Parkinson apresentaram redução da massa muscular, da endurance e das forças musculares respiratórias, e da capacidade física, se comparado aos indivíduos saudáveis. Além disso, a força muscular expiratória e a endurance da musculatura respiratória influenciam a capacidade física medida pelo teste de caminhada de seis minutos, assim como a massa muscular influencia na endurance da musculatura respiratória nos pacientes com DP, indicando que quanto maior a força muscular expiratória e a endurance muscular respiratória, maior a capacidade física; e quanto maior a quantidade de massa muscular, maior a endurance muscular respiratória. Dessa maneira, constatamos a importância de submeter esses pacientes a um programa de intervenção fisioterapêutica adequado às suas limitações e à gravidade da doença, bem como a necessidade de avaliar a capacidade física e tornar o teste de caminhada de seis minutos um método padrão de avaliação na prática clínica dos pacientes com doença de Parkinson.

\section{Referências}

1. Haase DCBV, Machado DC, Oliveira JGD. Atuação da fisioterapia no paciente com doença de Parkinson. Fisioter Mov. 2008;21(1):79-85.

2. Umphred DA. Reabilitação neurológica. 5. ed. Elsevier: Rio de Janeiro; 2009.

3. Prado RA, Teixeira ALC, Langa CJSO, Egydio PRM, Izzo P. A influência dos exercícios resistidos no equilíbrio, mobilidade funcional e na qualidade de vida de idosas. 0 Mundo da Saúde. 2010;34(2):183-91.

4. Canning CG, Alison JA, Allen NE, Groeller H. Parkinson's disease: an investigation of exercise capacity, respiratory function, and gait. Arch Phys Med Rehabil. 1997;78(2):199207. doi:10.1016/S0003-9993(97)90264-1.
5. Alves LA, Coelho AC, Brunetto AF. Fisioterapia respiratória na doença de parkinson idiopática: relato de caso. Fisioter. Pesq. 2005;12(3):46-9.

6. Mehanna R, Jankovic J. Respiratory problems in neurologic movement disorders. Parkinsonism Relat Disord. 2010;16(10):628-38.

7. Sathyaprabha TN, Kapavarapu PK, Pal PK, Thennarasu K, Raju TR. Pulmonary functions in Parkinson's disease. Indian J Chest Dis Allied Sci. 2005;47(4):251-7. PMid:16255396.

8. Pitts T, Bolser D, Rosenbek J, Troche M, Sapienza C. Voluntary cough production and swallow dysfunction in parkinson's disease dysphagia. 2008; 23(3):297-301.

9. Gorzoni ML, Russo MR. O envelhecimento respiratório. In: Freitas EV, Py L, Neri AL, Cançado FAX, Gorzoni ML, Rocha SM, organizadores. Tratado de Geriatria e Gerontologia. Rio de Janeiro: Guanabara Koogan; 2002. p. 340-343.

10. Rebelatto JR, Morelli JGS. Fisioterapia geriátrica: a prática da assistência ao idoso. 2. ed. Barueri São Paulo: Manole; 2007.

11. Podsiadlo D, Richardson S. The time "Up e Go": a test of basic functional mobility for frail elderly persons. J Am Geriatr Soc. 1991;39(2):142-8. PMid:1991946.

12. Solway S, Brooks D, Lacasse Y, Thomas S. Qualitative systematic overview of the measurement properties of functional walk tests used in the cardiorespiratory domain. Chest. 2001;119(1):256-70. doi:10.1378/ chest.119.1.256.

13. American Thoracic Society (ATS). Committee on Proficiency Standards for Clinical Pulmonary Function Laboratories. ATS statement: guidelines for the six-minute walk test. Am J Respir Crit Care Med. 2002; 166(1):111-7. PMid:12091180.

14. Casas A, Vilaro J, Rabinovich R, Mayer A, Barberà JA, Rodriguez-Roisin R, et al. Encouraged 6-min walking test indicates maximum sustainable exercise in COPD patients. Chest. 2005;128(1):55-61. doi:10.1378/ chest.128.1.55.

15. Leung ASY, Chan KK, Sykes K, Chan KS. Reliability, validity, and responsiveness of a 2 -min walk test to assess exercise capacity of COPD patients. Chest. 2006;130(1):119-25. doi:10.1378/chest.130.1.119. 
16. Goulart F, Pereira LX. Uso de escalas para avaliação da doença de Parkinson em fisioterapia. Fisioter Pesq. 2005;11(1):49-56.

17. Matsudo S, Araújo T, Matsudo V, Matsudo VKR, Andrade DR, Andrade EL, et al. Questionário Internacional de Atividade Física (IPAQ): estudo de validade e reprodutibilidade no Brasil. Rev Bras Ativ Saude. 2001;10:5-18.

18. Miller MR, Hankinson J, Brusasco V, Burgos F, Casaburi $\mathrm{R}$, Coates A, et al. ATS/ERS Task force: standardisation of lung function testing. Eur Respir J. 2005;26:319-38. doi:10.1183/09031936.05.00034805.

19. Mendes CCT, Raele R. Avaliação corporal por bioimpedância. Rev Nutr Pauta. 1997;24:12-4.

20. Vermeeren MA, Creutzberg EC, Schols AM, Postma DS, Pieters WR, Roldaan AC, et al. Prevalence of nutritional depletion in a large out-patient population of patients with COPD. Respiratory Med. 2006;100(8):1349-55. doi:10.1016/j.rmed.2005.11.023.

21. Black LF, Hyatt RE. Maximal respiratory pressures: normal values and relationship to age and sex. Am Rev Respir Dis. 1969;99(5):696-702. PMid:5772056.

22. Iwama AM, Andrade GN, Shima P, Tanni SE, Godoy I, Dourado VZ. The sixminute walk test and body weight-walk distance product in healthy Brazilian subjects. Braz J Med Biol Res. 2009;42(11):1080-5. doi:10.1590/S0100-879X2009005000032.

23. Borg GA. Psychophysical bases of perceived exertion. Med Sci Sports Exerc. 1982;14(5):377-81.

24. Global Iniciative for Chronic Obstructive Lung Disease (GOLD). Global Strategy for the diagnosis, management, and prevention of chronic obstructive pulmonary disease. [cited 23 Nov. 2010]. Available at: http:// www.goldcopd.org/gold-around-the-world.html.

25. Rossi AP, Watson NL, Newman AB, Harris TB, Kritchevsky SB, Bauer DC, et al. Effects of body composition and adipose tissue distribution on respiratory function in elderly men and women: the health, aging, and body composition study. J Gerontol A Biol Sci Med Sci. 2011;66(7)801-8.

26. Sabate M, Gonzalez I, Ruperez F, Rodriguez M. Obstructive and restrictive pulmonary dysfunctions in Parkinson's disease. J Neurol Sci. 1996;138(1-2):1149. doi:10.1016/0022-510X(96)00003-2.
27. Lilker ES, Woolf CA. Pulmonary function in Parkinson's syndrome: the effect of thalotomy. Can Med Assoc J 1968;99(15):752-7. PMid:5700845.

28. Neu HC, Connolly JJ Jr, Schwertley FW, Ladwig HA, Brody AW. Obstructive respiratory dysfunction in parkinsonian patients. Am Rev Respir. Dis.1967;95(1): 33-47. PMid:6015923.

29. Obenour WH, Stevens PM, Cohen AA, McCutchen JJ. The causes of abnormal pulmonary function in Parkinson's disease. Am Rev Respir Dis. 1972;105(3):382-7. PMid:5011667.

30. Brunetto AF, Pitta FO, Paulin E, Probst VS, Yamaguti WPS, Ferreira LF. Relação entre capacidade ventilatória e exercício em pacientes com doença pulmonar obstrutiva crônica moderada-grave. Rev Bras Fisioter. 2003;7(1):41-7.

31. Pitta F, Troosters T, Spruit MA, Probst VS, Decramer M, Gosselink R. Characteristics of physical activities in daily life in chronic obstructive pulmonary disease. Am J Respir Crit Care Med. 2005;171(9):972-7. doi:10.1164/rccm.200407-8550C.

32. Troosters T, Gosselink R, Decramer M. Six minute walking distance in healthy elderly subjects. Eur Respir J. 1999;14(2):270-4. doi:10.1034/ j.13993003.1999.14b06.x.

33. Gosselink R, Troosters T, Decramer M. Peripheral muscle weakness contributes to exercise limitation in COPD. Am J Respir Crit Care Med. 1996; 153(3):97680. PMid:8630582.

34. American Thoracic Society and European Respiratory Society. Skeletal muscle dysfunction in chronic obstructive pulmonary disease. Am J Respir Crit Care Med. 1999;159 (4 Pt 2):S1-40. PMid:9872811.

35. Bernard S, LeBlanc P, Whitton F, Carrier G, Jobin J, Belleau R, et al. Peripheral muscle weakness in patients with chronic obstructive pulmonary disease. Am. J Respir Crit Care Med. 1998;158(2):629-34. PMid:9700144.

36. Ferreira IM, Brooks D, Lacasse Y, Goldstein RS, White J. Nutritional supplementation for stable chronic obstructive pulmonary disease. Cochrane Database Syst Rev. 2005;18(2). PMCid:1087867.

37. Falvo MJ, Earhart GM. Six-minute walk distance in persons with parkinson disease: a hierarchical regression model. Arch Phys Med Rehabil. 2009;90(6):1004-8. 
38. Cestaro EJ, Lorenzo VAPD, Marino DM, Walsh I, Ruas G, Jamami M, et al. Fatores que influenciam a capacidade física de pacientes com doença pulmonar obstrutiva crônica. Fisioter Pesq. 2010;17(4):332-6.

39. Celli BR, Cote CG, Marin JM, Casanova C, Montes de Oca M, Mendez RA, et al. The body mass index, airflow obstruction, dyspnea, and exercise capacity index in chronic obstructive pulmonary disease. N Engl J Med. 2004;350:1005-12. doi:10.1056/NEJMoa021322.
40. Cahalin LP, Mathier MA, Semigran MJ, Dec GW, DiSalvo TG. The six-minute walk test predicts peak oxygen uptake and survival in patients with advanced heart failure. Chest. 1996;110(2):325-32. doi:10.1378/ chest.110.2.325.

Recebido: 19/08/2011 Received: 08/19/2011

Aprovado: $11 / 04 / 2012$

Approved: 04/11/2012 